\title{
MODELS FOR THE FORMATION OF BINARY AND MILLISECOND PULSARS
}

\author{
D. BHATTACHARYA \\ Raman Research Institute \\ Bangalore 560080. India.
}

\section{Introduction}

Under the name "binary and millisecond pulsars" are grouped a number of radio pulsars, forming roughly $10 \%$ of the known pulsar population, which distinguish themselves on three counts:

- Short spin period

- Low magnetic-field strength

- Presence of an evolved binary companion

Although in a few cases not all the three characteristics are present, this class brings together those pulsars whose spin history has involved a stage of spin up on accretion of matter from a binary companion, a process popularly known as "recycling". Another name of this group of pulsars is thus "recycled pulsars".

Understanding the formation of these pulsars mainly concerns understanding each of the above characteristics - namely (i) why the spin periods of the majority of them are short, (ii) why do most of them have much lower magnetic fields than isolated pulsars (see Fig. 1), and (iii) what evolutionary path led to the specific orbital characteristics and companion masses that individual pulsars of this class have. In this article we shall mainly concentrate on the third issue, and make only a few remarks about the first two.

\section{Spin Periods}

It is quite remarkable that most pulsars with binary companions have very short spin periods (see Fig. 1). As mentioned above, this is attributed to the 


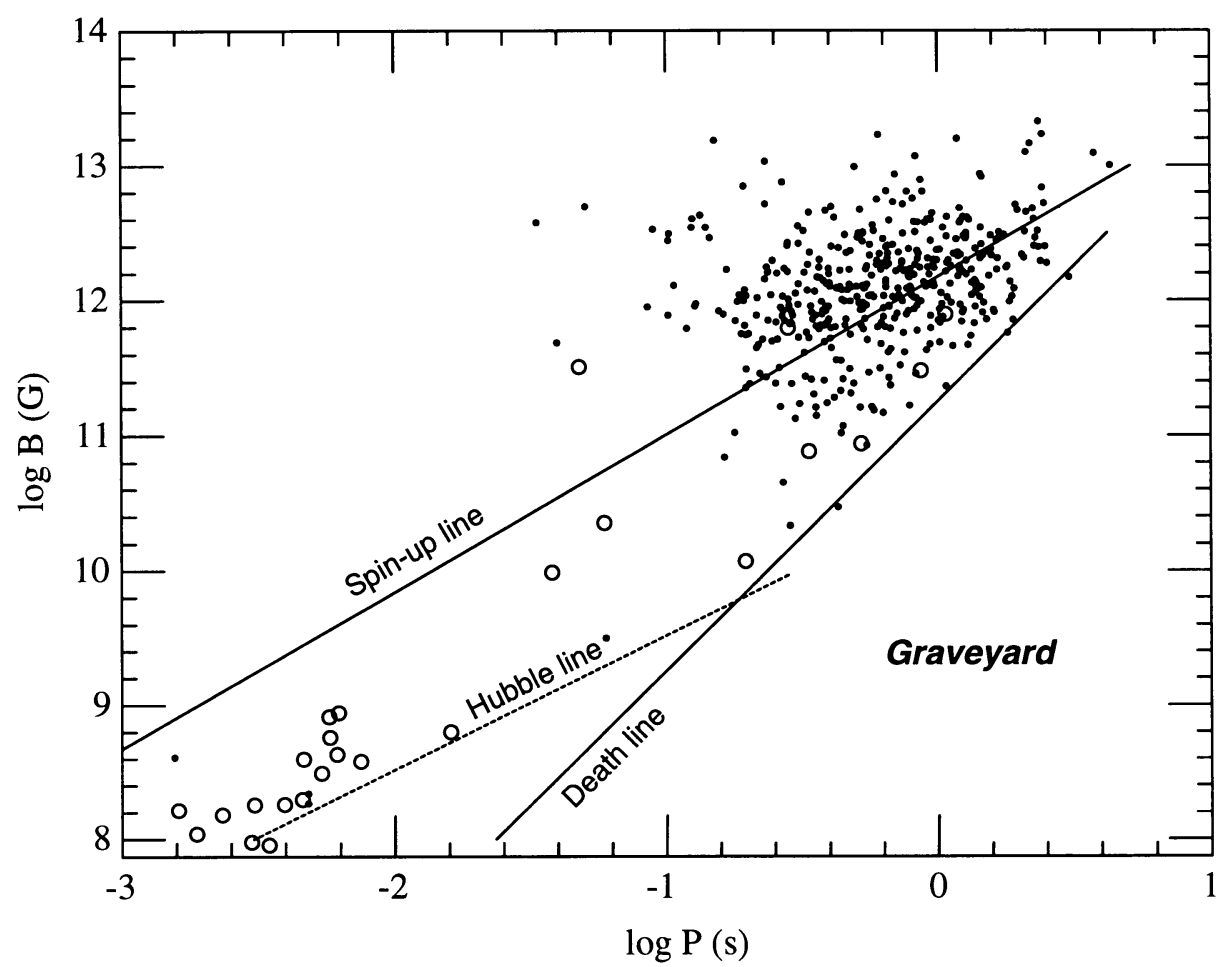

Figure 1. The magnetic fields and periods of known pulsars in the galactic disc. Filled dots indicate isolated pulsars and open circles binaries. The spin up line is the relation defined in eq. (1), with $\dot{M}=\dot{M}_{\mathrm{Edd}}$. Pulsar activity ceases to the right of the death line, and the spin down age $(\equiv P / 2 \dot{P})$ of a pulsar equals $10^{10} \mathrm{yr}$ on the Hubble line.

spin up of the neutron star during accretion, as is seen to be happening in a number of $\mathrm{X}$-ray binaries. A rough description of this process is as follows. During the mass transfer from the companion to the magnetized neutron star, matter approaches the neutron star through an accretion disc, in quasiKeplerian orbits. As it approaches the "magnetospheric radius" $r_{\mathrm{m}}$ of the neutron star, the dynamics of the matter flow comes to be dominated by the magnetic field of the neutron star, and the matter is forced to corotate with the neutron star. If the corotation speed exceeds Keplerian speed at this point, the neutron star loses spin angular momentum to the incoming matter, and slows down. If, on the other hand, the Keplerian speed exceeds the corotation speed, part of the orbital angular momentum of the matter is transferred to the neutron star till corotation is achieved, spinning the neutron star up as a result. Given enough time a spin equilibrium would be achieved, where the Keplerian speed and the corotation speed would match 
at the magnetospheric radius. This leads to an "equilibrium spin period"

$$
P_{\text {eq }}=1.9 \mathrm{~ms}\left(\frac{B_{\mathrm{s}}}{10^{9} \mathrm{G}}\right)^{6 / 7}\left(\frac{\dot{M}}{\dot{M}_{\text {Edd }}}\right)^{-3 / 7}
$$

where $B_{\mathrm{s}}$ is the dipole field strength at the surface of the neutron star, $\dot{M}$ is the rate of mass accretion and $\dot{M}_{\text {Edd }}$ is the Eddington accretion rate. From the rate of accretion of angular momentum, it can also be estimated that spin up to $P_{\text {eq }}$, starting from a much longer spin period, would require the accretion of a minimum mass

$$
\Delta M \simeq 0.1 \mathrm{M}_{\odot}\left(P_{\text {eq }} / 1.5 \mathrm{~ms}\right)^{-4 / 3}
$$

Admittedly, the picture described above is rather simplified (see Ghosh, these proceedings, for a more detailed discussion), but it does produce correct order-of-magnitude estimates.

One of the successes of this spin up (recycling) hypothesis for the origin of short spin periods of binary and millisecond pulsars is that most of these pulsars lie to the right of the critical "spin up line" in the $(B-P)$ diagram (Fig. 1), obtained by setting $\dot{M}=\dot{M}_{\text {Edd }}$ (the maximum accretion rate) in Eq. (1). An important point to note is that if a neutron star has to be spun up to a period as short as a few milliseconds in an Eddingtonlimited accretion, it would take a persistent accretion phase lasting $\sim 10^{7} \mathrm{yr}$ or more, since the Eddington limit for a neutron star is $\sim 10^{-8} \mathrm{M}_{\odot} \mathrm{yr}^{-1}$. In addition, it would also need the magnetic field of the neutron star to be low, $\lesssim 10^{9} \mathrm{G}$.

\section{Magnetic-Field Strength}

Most pulsars processed in interacting binaries have considerably lower field strengths than garden-variety isolated pulsars (Fig. 1). The reason for this is not very well understood, but several physical models have been proposed. For about two decades following the discovery of pulsars it was believed that the magnetic fields of neutron stars undergo spontaneous Ohmic decay with a time scale of a few million years. In this picture, the recycled pulsars would have lower magnetic-field strengths simply because they are older. For a variety of reasons, however, this is no longer a preferred hypothesis [see Bhattacharya \& Srinivasan (1995) for a review]. The idea that processing of a neutron star in a binary system is directly responsible for lowering its field strength (Bisnovatyi-Kogan \& Komberg 1974; Taam \& Van den Heuvel 1986; Bailes 1989) has of late gained currency. Physical models proposed for this kind of field evolution fall in two major categories, namely (a) Spindown-induced field evolution, and (b) Mass accretion-induced field evolution. 
Models of category (a) propose an intimate relation between the spin period of the star and its magnetic field strength. At least two different physical mechanisms have been considered to accomplish this. One suggestion involves the interaction of the Abrikosov fluxoids in the superconducting protons carrying the magnetic flux in the neutron star core with the quantized neutron superfluid vortices which carry the angular momentum. Spin down (prior to spin up due to accretion) of the neutron star causes neutron vortices to move outwards, carrying the fluxoids with them. This "expelled" flux then undergoes Ohmic decay in the crust. Srinivasan et al. (1990) argued that this manner of evolution is in good qualitative agreement with observed distribution of isolated and binary pulsar fields. Quantitative modelling of low-mass binary systems in this scenario has also yielded encouraging results (Jahan Miri \& Bhattacharya 1994). The second type of models in this category associate magnetic field evolution with crustal plate tectonics (Ruderman 1991). According to this picture spin down causes plates with frozen magnetic field configurations to move towards the stellar equator, where opposite poles can combine and destroy much of the magnetic field.

Category (b) is an even larger mixed bag of models, but practically all of them assume an initial field configuration confined entirely to the outer crust of the neutron star. The physical effects proposed here have been (i) screening of the stellar magnetic field by incoming diamagnetic plasma (Bisnovatyi-Kogan \& Komberg 1974), (ii) an inverse thermoelectric battery that destroys existing field as heat flows up the density gradient (Blondin \& Freese 1986), (iii) advection of the field with the accreted matter into the deeper layers of the crust, where the plasma expands sideways and reconnection occurs (Romani 1990), (iv) reduction of crustal conductivity due to heating resulting from accretion and consequent enhanced ohmic decay of the magnetic field (Urpin \& Geppert 1994) and (v) compression of the current-carrying layers causing reduction in length scale of the field distribution and hence faster Ohmic decay (Konar et al. 1994). These models require further development before direct comparison with observations can be made.

In short, a number of models have been proposed, and are being currently worked upon, which attempt to explain the low magnetic fields of recycled pulsars as a direct result of the interaction of the neutron star with its binary companions. However at present we have neither sufficient knowledge about these models, nor enough observational constraints to unequivocally choose between them. 


\section{Orbital Characteristics}

We now come to the major part of this paper, namely the discussion of the orbital characteristics of binary and millisecond pulsars, the nature of their companions, and the probable evolutionary history leading up to the present systems.

Binary and millisecond pulsars come with a great variety of companion types, which can be roughly classified into the following categories:

1. None

2. Evaporating secondary with very low mass

3. White dwarf with mass $<0.45 M_{\odot}$

4. White dwarf with mass $>0.45 M_{\odot}$

5. Neutron star

6. Massive main-sequence star

7. Low-mass main-sequence star (?)

8. Planets

Of these, pulsars with companion types 1-3 above are called low-mass binary pulsars (LMBPs), and those with companion types 4 and 5 highmass binary pulsars (HMBPs). Companions of type 6 and 7 are unevolved, the pulsars are therefore not "recycled", and will hence be excluded from the present discussions. One millisecond pulsar in the galactic disc (PSR 1257+12, see Wolszczan, these proceedings) and perhaps one in a globular cluster (PSR 1620-26, Thorsett et al. 1993) have been reported to have planetarysized companions. It is not clear, however, whether they belong to the low-mass or the high-mass class (see Section 7).

What are the progenitors of these pulsars? Since they have undergone mass accretion at some point in their lives, clearly their progenitors are to be looked for among the accreting binaries with neutron star components. Two major classes of such binaries are known - the high-mass X-ray binaries (HMXBs), and the low-mass X-ray binaries (LXMBs). The low-mass binary pulsars are usually thought to have descended from low-mass X-ray binaries, and the high-mass binary pulsars from high-mass $\mathrm{X}$-ray binaries.

According to the conventional classification, a low-mass $\mathrm{X}$-ray binary consists of a neutron star and a donor star with mass less than the mass of the neutron star (although Her X-1 with a donor mass $\sim 2 \mathrm{M}_{\odot}$ is also usually grouped with this category). The traditional HMXBs, on the other hand, contain donors more massive than $\sim 8 \mathrm{M}_{\odot}$. There is a clear gap in the donor masses between these two categories - the "intermediate-mass" secondaries do not seem to be represented among the visible $\mathrm{X}$-ray binaries. This does not mean, however, that binaries containing intermediate-mass companions to neutron stars do not exist in nature. Our failure to detect them may be caused by a very short $\mathrm{X}$-ray lifetime of these objects. It is well known 
that the Roche-lobe overflow mass transfer is unstable if the donor mass exceeds that of the neutron star, and this phase is probably marked by a shrouding of the accreting $\mathrm{X}$-ray source by a large quantity of outflowing matter, keeping $\mathrm{X}$-rays out of view. This is also true of high-mass binaries, of course - but high-mass stars have strong stellar winds, sufficient to generate detectable X-rays in HMXBs before the Roche-lobe overflow occurs. In intermediate mass binaries the weak stellar winds are unlikely to give rise to a detectable accreting X-ray source. We must therefore be aware that the intermediate-mass binaries, as a class, may well exist and contribute to recycled pulsar population, although they may not be represented among the accreting $\mathrm{X}$-ray binaries. We shall have more to say about this below.

\section{The Evolution of High-Mass X-ray Binaries and the Origin of HMBPs}

According to the standard evolutionary scenario, high-mass X-ray binaries produce $\mathrm{X}$-rays in the two early phases - when the neutron star accretes the stellar wind of the companion, and when only the atmosphere of the donor overflows the Roche lobe. Beyond this point, very heavy mass transfer due to Roche-lobe overflow ensues and the neutron star spirals into the massive secondary. If the initial orbital period is less than $\sim 1$ year, a complete spiral-in is likely, with the neutron star entering the centre of the massive companion, and a Thorne- $\dot{Z} y t k o w$ object is produced. The final outcome of this is unclear - if the Thorne-Żytkow object is able to lose most of its envelope either in stellar winds or due to magneto-rotational effects a recycled pulsar may be left, otherwise a black hole appears to be the inevitable result (see Podsiadlowski, these proceedings).

If the initial orbit is wide enough, with orbital period larger than $\sim 1$ year, then according to the conventional picture the neutron star would escape complete spiral in, and will be left in a tight binary with the helium core of the secondary. This helium star, if it is massive enough, would in time explode in a supernova leaving a new-born neutron star. If the binary survives the explosion, one expects to see a recycled pulsar and a young neutron star in an eccentric orbit, similar to PSR $1913+16$ and PSR 1534+12 systems. On the other hand if the binary disrupts the final result would be an isolated young pulsar and an isolated recycled pulsar. If the helium star is not massive enough to produce a neutron star, it would expand and transfer mass to the neutron star companion (lighter helium stars expand more). A second spiral-in may occur and eventually a recycled pulsar and a heavy white dwarf in a compact, circular orbit will be left similar to the observed system PSR 0655+64.

While the above scenario appears reasonable for the production of dou- 
TABLE 1. HMBP orbital periods

\begin{tabular}{lcc}
\hline Pulsar Name & Binary components & Orbital period \\
\hline B1913+16 & NS + NS & $8 \mathrm{~h}$ \\
B1534+12 & NS + NS & $10 \mathrm{~h}$ \\
B2303+46 & NS + NS & $12 \mathrm{~d}$ \\
B0655+64 & NS + WD & $1 \mathrm{~d}$ \\
J1023+10 & NS + WD & $8 \mathrm{~d}$ \\
J2145-0750 & NS + WD & $7 \mathrm{~d}$ \\
\hline
\end{tabular}

NS $=$ Neutron Star WD $=$ White Dwarf

ble neutron star and neutron star + heavy white-dwarf binaries, it does depend heavily on the poorly understood evolutionary phase of spiral-in. Recently, Chevalier (1993) and Brown (1994) have questioned the scenario on this ground. These authors show that in the case of a spherically symmetric mass transfer, a neutron star spiralling into a hydrogen-rich giant would experience a "hypercritical accretion", with transfer rates exceeding $0.1 \mathrm{M}_{\odot} \mathrm{yr}^{-1}$, when the Eddington limit is no longer valid since most of the cooling is done by neutrinos. Such a neutron star will end up accreting an enormous quantity of material and would invariably collapse to a black hole. If this assertion proves to be correct, then one can never hope to obtain a double neutron star system or a compact neutron star-white dwarf binary through the conventional spiral-in.

Indications from the observations are, however, that spiral-in products do exist. Systems listed in Table 1 all have short binary periods, and rather heavy compact remnants - the initial orbit of the progenitor system must have been considerably wider, since the present orbits are not wide enough to accommodate even one of the progenitor stars in the main sequence or a slightly evolved phase.

What does the existence of these systems tell us? Either, the manifestly non-spherical geometry of the spiral-in process manages somehow to avoid the predicament of hypercritical accretion [although both Chevalier (1993) and Brown (1994) argue that taking angular momentum into consideration should not change their result], or one must find their progenitors among hitherto unconsidered class of objects. Brown (1994) suggests that double helium star binaries might be the progenitors of some double neutron star binaries. Finding suitable progenitors of neutron star + white-dwarf binaries might be even more difficult. Clearly this question deserves careful attention and further study. 


\section{Low-Mass X-ray Binaries and the Origin of LMBPs}

The standard evolution of low mass X-ray binaries follows one of two classical tracks, depending on the starting orbital period $P_{0}$ : for $P_{0} \lesssim 0.5$ day the evolution is driven by angular momentum loss by gravitational radiation and magnetic braking. These systems come into contact while the donor is on the main sequence and continues to shrink as mass transfer proceeds. The orbital period is expected to pass through a minimum of $\sim 80 \mathrm{~min}$, at which point the secondary would become degenerate and the orbit would widen again, albeit very slowly. It seems likely that the full course of this evolution is never followed, and at quite an early stage irradiation of the companion might significantly modify the evolutionary track (Podsiadlowski 1991; Harpaz \& Rappaport 1991; Tavani 1991). The nature and extent of this modification is, however, hotly debated (see Ritter, these proceedings), and a clear picture is yet to emerge.

Evolution of LMXBs with wider initial orbits $\left(P_{0} \gtrsim\right.$ a few days $)$ seems to be better understood - nuclear evolution drives the expansion of the secondary and hence the mass transfer, and the orbit widens as a result. Typically an increase of orbital period by about an order of magnitude is expected between the beginning and the end of the mass transfer (Webbink, Rappaport \& Savonije 1983). The mass transfer ends when the white-dwarf core of the donor is left. This evolutionary sequence leaves a helium white dwarf, with a mass $\leq 0.45 \mathrm{M}_{\odot}$, orbiting the recycled pulsar in a circular orbit. The wider the initial orbital period, the more evolved is the donor at the time of contact and hence the heavier is the final white dwarf remnant. This defines a relation between the final secondary mass and the final orbital period (Joss, Rappaport \& Lewis 1987), and most low-mass binary pulsars seem to obey this relationship, within observational uncertainties, strengthening the case for their origin from LMXBs [see Phinney \& Kulkarni (1994) and Lorimer (1994) for a comparison of the observed data with the predicted relation]. It must, however, be pointed out that it has not yet been possible to fully reconcile the birth statistics of LMXBs and LMBPs of different categories (see Lorimer, these proceedings).

A curious point regarding the orbital-period distribution of LMBPs has been noted by Camilo (1994): there appears to be a "gap" in the period distribution between $12 \mathrm{~d}$ and $65 \mathrm{~d}$ where no LMBP is seen (see Fig. 2). No known selection effect would create such a gap - so if the gap is real and not a statistical fluke the reason behind it must be evolutionary. However, there is no known evolutionary effect leading to gaps in this range, either. Enhanced magnetic braking effects for LMXBs with initial orbital periods up to a few days have been considered (Pylyser \& Savonije 1988), and might produce a similar gap between $\sim 1-10$ days in the final orbital period, which 


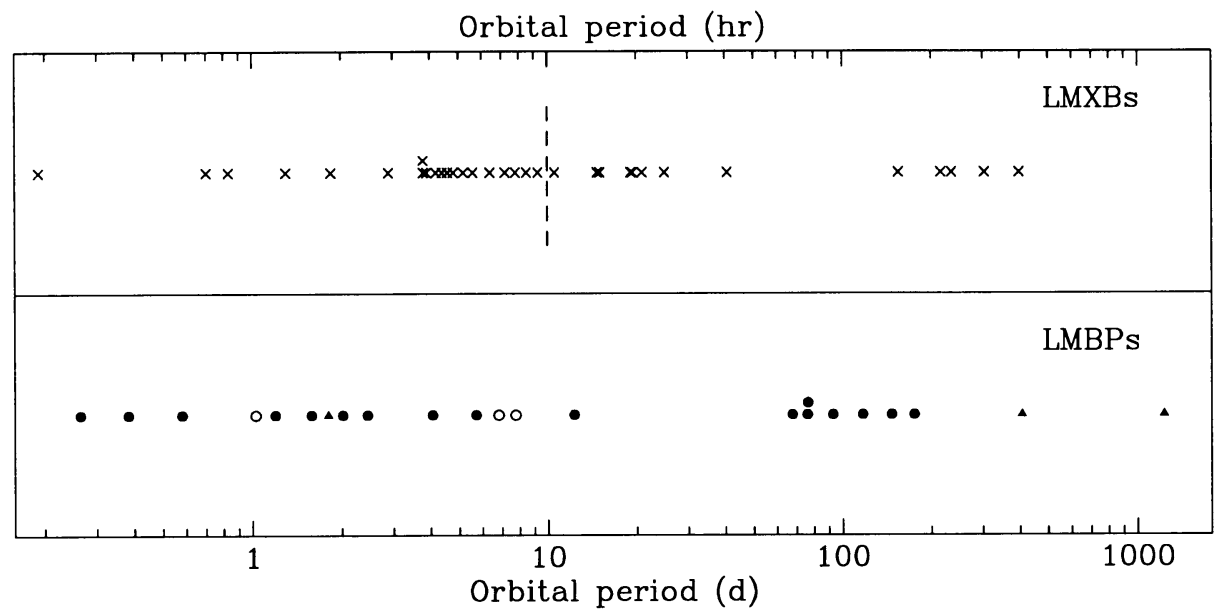

Figure 2. The distribution of the orbital periods of known low-mass X-ray binaries (top) and low-mass binary pulsars (bottom). Note the difference in scale between the two panels. Evolution of the systems to the left of the vertical dashed line in the top panel is strongly affected by angular momentum loss and the secondary comes into contact in the main sequence. Systems with larger orbital periods are likely to have evolved donors. In the bottom panel filled symbols are systems with secondary mass $\lesssim 0.45 \mathrm{M}_{\odot}$, while the open symbols denote systems with higher secondary masses, which are probable descendants of intermediate-mass binaries. Systems with eccentricity higher than 0.001 are shown as triangles. There appears to be a "gap" in the LMBP period distribution between 12 and 65 days. It may be that this is a reflection of a corresponding gap in the period distribution of LMXBs, in the range between $40 \mathrm{~h}$ and $5 \mathrm{~d}$, as seen in the top panel. (Figure courtesy Fernando Camilo.)

is still much smaller than the gap periods referred to above. On the other hand, this observed gap might reflect the original period distribution of LMXBs - indeed among the observed LMXBs there seems to be a dearth of systems with orbital periods between $40 \mathrm{~h}$ and 5 days (Fig. 2) - roughly the same range that might evolve to the region of the observed gap in the final evolutionary products.

\section{Millisecond Pulsars}

Millisecond pulsars, right since their discovery, have been considered to be products of LMXB evolution (Alpar et al. 1982). This is because their ultra-rapid spin $(P \lesssim 10 \mathrm{~ms})$ can be obtained by recycling only if the nearEddington accretion phase is prolonged - something that obtains only in LMXBs, particularly in wide ones. It is no surprise, therefore, that $\sim 75 \%$ of the known disc population of millisecond pulsars are LMBPs. What is surprising, however, is that quite a few millisecond pulsars are single, i.e., 
with no companion. It would be fair to say that we do not really understand the formation of single millisecond pulsars. If, like other millisecond pulsars, these too were formed in LMXBs then their companions had to be got rid of in some way. The foremost suggestion in this regard has been that of Ruderman et al. (1989), who argued that the radiation from a fastspinning millisecond pulsar can ablate its companion away, thus predicting the existence of systems like PSR $1957+20$ with evaporating secondaries. It appears, however, that the vaporization of the companion of $1957+20$ may not be vigorous enough to completely destroy it (Fruchter et al. 1990; Ryba \& Taylor 1991), and this pulsar being amongst the most powerful millisecond pulsars known casts some doubt on the effectiveness of this mechanism. However, a recently proposed variant of this scenario, involving irradiation and tidal dissipation, has been argued to be capable of destroying the companion of PSR $1957+20$ within $\sim 10^{8} \mathrm{yr}$ (Applegate \& Shaham 1994).

A second suggestion has been that the degenerate secondary, towards the end of the evolution of "close" LMXBs, might undergo unstable Roche lobe overflow as it expands on loss of mass, and be completely disrupted to form a heavy disc around the neutron star (Ruderman \& Shaham 1985). A serious objection to this idea was raised by Jeffrey (1986), who pointed out that even if such a disc were to form, slow accretion from it onto the neutron star would spin the neutron star down to a very long period well before the disc disappears.

Another way of losing the companion of a neutron star is the "ionization" of a binary due to an encounter with a third passing star. This may happen at times in globular clusters (see Rappaport, Putney \& Verbunt 1989), but would be an extremely rare occurrence in the galactic disc.

If these models are found to be inadequate to explain the occurrence of single millisecond pulsars, one must look for their origin outside the standard LMXB group. A view taken by some is that single millisecond pulsars are just born with short periods and low magnetic fields, and one need not invoke recycling to explain their origin (Michel 1987). While it can never be conclusively proven to be otherwise, it would seem odd that the single millisecond pulsars blend in very well with the rest of the millisecond pulsar population, which are clearly recycled, given their binary nature. Moreover, none of the millisecond pulsars in the galactic disc, including the single ones, lies above the "spin up line" in Fig. 1.

It has been pointed out by Bonsema \& Van den Heuvel (1985) that the merger of a white dwarf of mass exceeding $\sim 0.66 \mathrm{M}_{\odot}$ with a neutron star may well produce a millisecond pulsar and leave no companion for it. This remains a viable route, although the statistics of it are yet to be worked out. The progenitor in this case would be very similar to PSR $0655+64$, but with a tighter orbit (orbital period of a few hours). This kind of progenitor 
system would, in turn, be a descendant of a high-mass X-ray binary, or perhaps an intermediate-mass one.

An even more exotic scenario involving high-mass binaries would envisage the formation of a single millisecond pulsar as the end product of a Thorne-Żytkow object (Wijers et al. 1992). The degree of spin up that can occur during and after the spiral-in of the neutron star into the companion's envelope is not entirely clear. In case of a complete spiral-in, a fraction of the orbital angular momentum resides in the stellar envelope so one expects the Thorne-Żytkow stars to be rapidly spinning. Whether or not accretion of some of this material onto the central neutron star, the magnetic field of which might have been considerably weakened during the course of the evolution, can spin it up to millisecond periods needs to be quantitatively worked out.

At this point it is appropriate to state that every scenario for the formation of single millisecond pulsars that I described above, with the exception of that involving ionization, has also been used in the context of formation of planets around millisecond pulsars (see Podsiadlowski 1993 for a review). The generic idea in these models is that once the secondary is destroyed, its debris can eventually condense into planets. As a result, the situation is now slightly confused - it is not possible to tell which of these routes might really contribute to planet formation and which might leave genuinely isolated millisecond pulsars.

\section{Millisecond Pulsars from Intermediate-Mass Binaries}

To conclude this discussion, I would like to draw the attention of the reader to two recently discovered near-millisecond pulsars - PSR 2145-0750 and PSR 1023+10. Both have spin periods of $16 \mathrm{~ms}$. The orbital periods are short, 6.8 days and 7.8 days, respectively. Most importantly, the masses of their white-dwarf companions are moderately high $-0.5 \mathrm{M}_{\odot}$ and $0.8 \mathrm{M}_{\odot}$, respectively. Since helium white dwarfs cannot grow to masses beyond $0.45 \mathrm{M}_{\odot}$, these remnants must be $\mathrm{C}-\mathrm{O}$ dwarfs. This means that the progenitors of these remnants must have gone through helium burning, and hence mass transfer must have taken place in a rather late (AGB) phase - implying a rather wide initial orbit and a subsequent spiral in (Van den Heuvel 1994). According to the calculations of Tben \& Tutukov $(1985,1993)$ the original secondary masses in these cases should have been in the range 1-3 $\mathrm{M}_{\odot}$ for PSR 2145-0750 and 4-6 $\mathrm{M}_{\odot}$ for PSR 1023+10. For PSR 21450750 an original secondary mass towards the heavier end of the above range seems preferable, since PSR $0820+02$, starting with a donor of $\sim 1 \mathrm{M}_{\odot}$ and an orbital period of $\gtrsim 200$ days has expanded its orbit to the present period of 1232 days, and has left a $\sim 0.3-0.4 \mathrm{M}_{\odot}$ helium white-dwarf remnant. It 
seems likely, therefore, that the spiral-in products like PSR 2145-0750 and PSR $1023+10$ descend from systems with secondary masses higher than $\sim 1 \mathrm{M}_{\odot}$.

The important point that emerges from this is that these pulsars seem to be descendants of intermediate-mass binaries, with secondaries in the range 3-6 $\mathrm{M}_{\odot}$ (see also Kulkarni 1994). Indeed the pulsar PSR 0655+64 should also be classified in this category (Van den Heuvel \& Taam 1984). However, these two new pulsars have demonstrated that spin up in this class of binaries can proceed to very nearly the millisecond range. This could be the result of an effect pointed out by Iben \& Tutukov (1993) that the post-spiral-in remnants of stars with original mass in the range 4-6 $M_{\odot}$ continue to undergo Roche lobe overflow and transfer mass at the rate of $10^{-8}$ to $10^{-6} \mathrm{M}_{\odot} \mathrm{yr}^{-1}$ for about $10^{6} \mathrm{yr}$ (see also Van den Heuvel 1994).

In sum, these recent discoveries have established intermediate-mass binaries as substantial contributors to recycled pulsar population - and shown that many of these pulsars could be spinning very rapidly, at nearly millisecond periods. Intermediate-mass binaries have remained in relative oblivion so far because they do not show up as X-ray sources. These recycled pulsars have thus opened up a new and exciting avenue for the study of this class of binary systems.

Acknowledgements. I am indebted to Ed van den Heuvel, Philipp Podsiadlowski, Fernando Camilo and Duncan Lorimer for many discussions and access to unpublished information during the preparation of this talk, much of which was done during a very useful month spent at the Nuffield Radio Astronomy Laboratories at Jodrell Bank. A travel grant by the International Astronomical Union and hospitality of the University of Utrecht are gratefully acknowledged. 


\section{References}

Alpar, M.A., Cheng, A.F., Ruderman, M.A. \& Shaham, J. 1982, Nat 300, 728

Applegate, J.H. \& Shaham, J. 1994, ApJ 436, 312

Bailes, M. 1989, ApJ 342, 917

Bhattacharya, D. \& Srinivasan, G. 1995, in $X$-ray Binaries, W.H.G. Lewin, J. van Paradijs \& E.P.J. van den Heuvel (Eds.), Cambridge University Press, (in press)

Bisnovatyi-Kogan, G.S. \& Komberg, B.V. 1974, SvA 18, 217

Blondin, J.M. \& Freese, K. 1986, Nat 323, 786

Bonsema, P.F.J. \& Van den Heuvel, E.P.J. 1985, A\&A 146, L3

Brown, G.E. 1994, ApJ (in press)

Camilo, F. 1994, in Lives of the Neutron Stars, M.A. Alpar, Ü. Kızlloğlu \& J. van Paradijs (Eds.), Kluwer Academic Publishers, (in press)

Chevalier, R. 1993, ApJ 411, L33

Fruchter, A.S., Berman, G., Bower, G. et al. 1990, ApJ 351, 642

Harpaz, A. \& Rappaport, S.A. 1991, ApJ 383, 739

Iben, I. \& Tutukov, A.V. 1985, ApJS 58, 661

Iben, I. \& Tutukov, A.V. 1993, ApJ 418, 343

Jahan Miri, M. \& Bhattacharya, D. 1994, MNRAS 269, 455

Jeffrey, L.C. 1986, Nat 319, 384

Joss, P.C., Rappaport, S.A. \& Lewis, W. 1987, ApJ 319, 180

Konar, S., Bhattacharya, D. \& Urpin, V.A. 1994, in Proceedings of the 6th Asia-Pacific Regional Meeting of the IAU, V.K. Kapahi \& N. Dadhich (Eds.), (in press)

Kulkarni, S.R. 1994, in: Proceedings of the Aspen Conference on Millisecond Pulsars: A Decade of Surprises, (in press)

Lorimer, D. 1994, The Galactic Population of Millisecond and Normal Pulsars, Ph.D. Thesis, University of Manchester

Michel, F.C. 1987, Nat 329, 310

Phinney, E.S. \& Kulkarni, S.R. 1994, ARA\&A (in press)

Podsiadlowski, P. 1991, Nat 350, 136.

Podsiadlowski, P. 1993, in: Planets Around Pulsars, J.A. Phillips, S.E. Thorsett \& S.R. Kulkarni (Eds.), Astron. Soc. of the Pacific (San Fransisco)

Pylyser, E.H.P. \& Savonije, G.J. 1988, A\&A 191, 57

Rappaport, S.A., Putney, A. \& Verbunt, F. 1989, ApJ 345, 210

Romani, R.W. 1990, Nat 347, 741

Ruderman, M. 1991, ApJ 366, 261

Ruderman, M. \& Shaham, J. 1985, ApJ 289, 244

Ruderman, M., Shaham, J. \& Tavani, M. 1989, ApJ 336, 507

Ryba, M.F. \& Taylor, J.H. 1991, ApJ 380, 557

Srinivasan, G., Bhattacharya, D., Muslimov, A.G. \& Tsygan, A.I. 1990, Curr. Sci. 59, 31

Taam, R.E. \& Van den Heuvel, E.P.J. 1986, ApJ 305, 235

Tavani, M. 1991, ApJ 366, L27

Thorsett, S.E., Arzoumanian, Z. \& Taylor, J.H. 1993, ApJ 412, L33

Urpin, V. \& Geppert, U. 1994, A\&A (submitted)

Van den Heuvel, E.P.J. 1994, A\&A (in press)

Van den Heuvel, E.P.J. \& Taam, R.E. 1984, Nat 309, 235

Webbink, R.F., Rappaport, S.A. \& Savonije, G.J. 1983, ApJ 270, 678

Wijers, R.A.M.J., Van den Heuvel, E.P.J., Van Kerkwijk, M.H. \& Bhattacharya, D. 1992, Nat 355, 593 


\section{Discussion}

S.R. Kulkarni: The period gap may be spurious, but I would like to draw your attention to a curious point. Pylyser \& Savonije (1988, A\&A 191,57 ) considered the evolution of compact LMXBs (accretion driven by angular momentum loss). Several authors (Taam, Webbink et al.) have considered the evolution of extended (Roche-lobe fed) LMXBs. These models disagree in the final orbital period range where they overlap (see Coté \& Pylser 1989, A\&A 218, 131). This suggests that in the final period range around about 10 days the evolution is extremely sensitive to the input physics. There could well be a discontinuity in this period range.

D. Bhattacharya: I agree that evolution dominated by angular momentum loss for short period binaries could be the cause of the period gap. However, even as strong an angular momentum loss considered by Pylyser and Savonije (1988) would be unable to place the gap where it is. Perhaps an even stronger angular momentum loss can do so. 